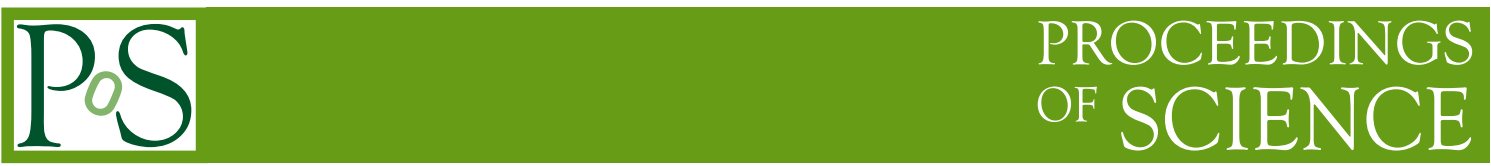

\title{
The ATLAS Trigger System - Ready for Run-2
}

\author{
Moritz Backes* \\ On behalf of the ATLAS Collaboration \\ CERN, Switzerland \\ E-mail: Moritz.Backesecern.ch
}

The ATLAS trigger system successfully collected collision data during the first run of the LHC between 2009-2013 at a centre-of-mass energy between $900 \mathrm{GeV}$ and $8 \mathrm{TeV}$. The trigger system consists of a hardware based Level-1 and a software based high-level trigger that reduces the event rate from the design bunch-crossing rate of $40 \mathrm{MHz}$ to an average recording rate of a few hundred Hz. With the increased centre-of-mass energy of $13 \mathrm{TeV}$ and the higher instantaneous luminosity expected during the Run- 2 data-taking campaign the rates of Run-1 trigger selections would rise by approximately a factor of 5 .

In these proceedings we briefly review the ATLAS trigger system upgrades that were implemented during the shutdown, allowing us to cope with the increased trigger rates while maintaining or even improving our efficiency to select relevant physics processes. This includes changes to the Level-1 calorimeter and muon trigger system, the introduction of a new Level-1 topological trigger subsystem and the merging of the previously two-level high-level trigger system into a single event filter farm. We show the performance improvements in the high-level trigger algorithms used to identify leptons, hadrons and global event quantities like missing transverse energy. Finally, we present the commissioning status of the trigger system and its initial performance in the 2015 run.

XXVII International Symposium on Lepton Photon Interactions at High Energies 17-22 August 2015

Ljubljana, Slovenia

* Speaker. 


\section{Introduction}

\footnotetext{
${ }^{1}$ In Run-2 the average number of collisions per proton-proton bunch crossing is expected to reach approximately 40-45 compared to values of 25-30 in Run-1.

${ }^{2}$ ATLAS uses a right-handed coordinate system with its origin at the nominal interaction point (IP) in the centre of the detector and the $z$-axis along the beam pipe. The $x$-axis points from the IP to the centre of the LHC ring, and the $y$-axis points upward. Cylindrical coordinates $(r, \phi)$ are used in the transverse plane, $\phi$ being the azimuthal angle around the beam pipe. The pseudorapidity is defined in terms of the polar angle $\theta$ as $\eta=-\ln \tan (\theta / 2)$.
} 


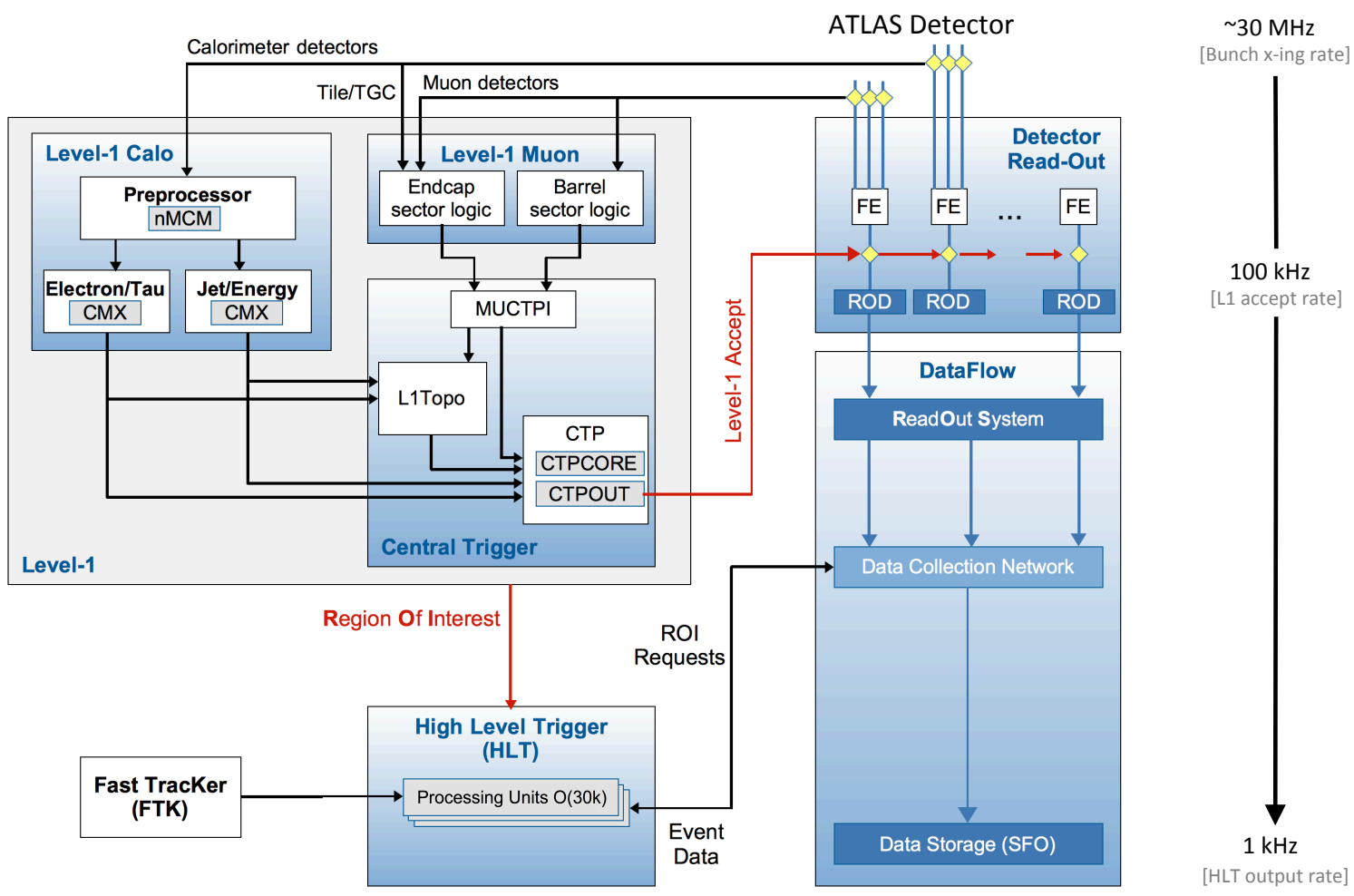

Figure 1: Schematic layout of the Run-2 ATLAS trigger and data acquisition sytem.

ger rates at L1 which were a major limitation in Run-1. In addition, the number of definable L1 calorimeter trigger thresholds, such as for example electromagnetic, tau, jet, or missing transverse energy thresholds, was doubled with respect to Run-1 and provides added flexibility for the trigger selections.

In the L1 muon system, information from the inner end-cap muon chambers, which are in front of the end-cap toroid magnets, and from the extended barrel region of the Tile calorimeter are used in timing coincidences with the L1 muon system information already in place from Run-1. These new coincidences are expected to significantly decrease the the L1 muon trigger rates in the forward region $(1.0<|\eta|<1.9)$ which are dominated by low- $p_{\mathrm{T}}$ out-of-time charged particles (e.g. protons) generated in the toroid magnet and the detector shielding. In addition, new trigger chambers in the feet of the detector barrel region are expected to bring a gain of approximately $4 \%$ in acceptance for L1 muons at $|\eta|<1.05$.

For Run-2 a conceptually new L1 topological sub-system (L1Topo) [3] is being put into place which performs topological selections based on L1 physics objects at the LHC bunch crossing rate. The L1Topo algorithms are implemented in FPGAs and form a decision within a latency of $200 \mathrm{~ns}$. Examples of possible topological selections are angular and invariant mass requirements, or global event quantities such as the sum of the transverse momenta of all L1 jet objects. Topological triggers are crucial for the Run-2 physics program as they allow the energy and momentum thresholds for multi-object final states to be kept low while significantly decreasing the trigger rates.

The information of all L1 sub-systems is combined in the CTP which forms the final L1 trigger decision. The CTP has undergone a major upgrade for Run-2 and is now able to accommodate 512 
L1 trigger thresholds (twice as many as in Run-1). In addition the CTP can now handle trigger rates of up to $100 \mathrm{kHz}$ which is a significant improvement over the $70 \mathrm{kHz}$ limit in Run-1.

The HLT has also undergone significant upgrades for Run-2. The most important structural change is the unification of the previously two-level system (Level-2 and Event Filter) into a single event processing farm which reduces the overall complexity of the system and allows for dynamic resource sharing between algorithms that were previously divided by being specific to one of the two HLT trigger levels.

In this way an efficient coupling of the HLT selection steps is achieved which reduces the duplication of CPU usage and network transfer of detector data. In addition, there has been a major redesign of the Run-1 trigger reconstruction algorithms to be closer to the offline reconstruction algorithms. The advantages of this approach are a large reduction in the duplication of online and offline software, an easier commissioning and calibration of the triggers along with offline selections and an increased physics acceptance after analysis selections. Furthermore, the HLT algorithms now have the capability to use real-time pile-up information to correct for effects of multiple interactions per bunch crossing.

\section{Commissioning and performance of the ATLAS Trigger system in early Run-2}

The upgraded ATLAS trigger system is currently being commissioned. In this section, the performance of the main physics triggers in the initial phase of Run-2 with 50 ns proton bunch spacing is discussed.

Fig. 2 (top left) shows the combined L1 and HLT efficiency of a single electron trigger (denoted HLT_e24_lhmedium_iloose_L1EM18VH) as a function of the electron candidate's transverse energy reconstructed 'offline' in the final data processing. The efficiency is measured with respect to these fully reconstructed electrons that are required to pass likelihood-based "lhmedium" identification. The trigger requires an electron candidate with $E_{\mathrm{T}}>24 \mathrm{GeV}$ satisfying the likelihood-based "lhmedium" identification criteria as well as a loose track isolation requirement. The HLT trigger is seeded by a level-1 trigger (L1_EM18VH) that applies an $E_{\mathrm{T}}$ dependent veto against energy deposited in the hadronic calorimeter behind the electron candidate's electromagnetic cluster. The efficiencies are measured with a tag-and-probe method using $Z \rightarrow e e$ decays with no background subtraction applied. They are compared to expectation from $Z \rightarrow e e$ simulation. The error bars show the statistical uncertainties only. Fig. 2 (top right) shows output rates of various single electron triggers as a function of the instantaneous luminosity in early Run-2. It can be seen that the rates for electron triggers with likelihood-based identification criteria ("Ihmedium" and "lhtight") are lower than those corresponding to triggers with cut-based identification selections ("medium" and "tight"). More details can be found in [reference PoS LP15 proceedings: "The Upgrade and Performance of the ATLAS Electron and Photon Triggers towards Run II" by Joseph Reichert].

Fig. 2 (middle left) shows the efficiency of a L1 single muon trigger (denoted L1_MU15) and the OR of the HLT_mu20_iloose_L1MU15 and HLT_mu50 high-level triggers as a function of the transverse momentum of offline muon candidates in the endcap detector region. The L1_MU15 trigger requires that a candidate passed the $15 \mathrm{GeV}$ threshold requirement of the $\mathrm{L} 1$ muon trigger system. The efficiency is computed relative to the offline muon candidates which are reconstructed using standard ATLAS software and are required to pass "medium" quality requirement. The 

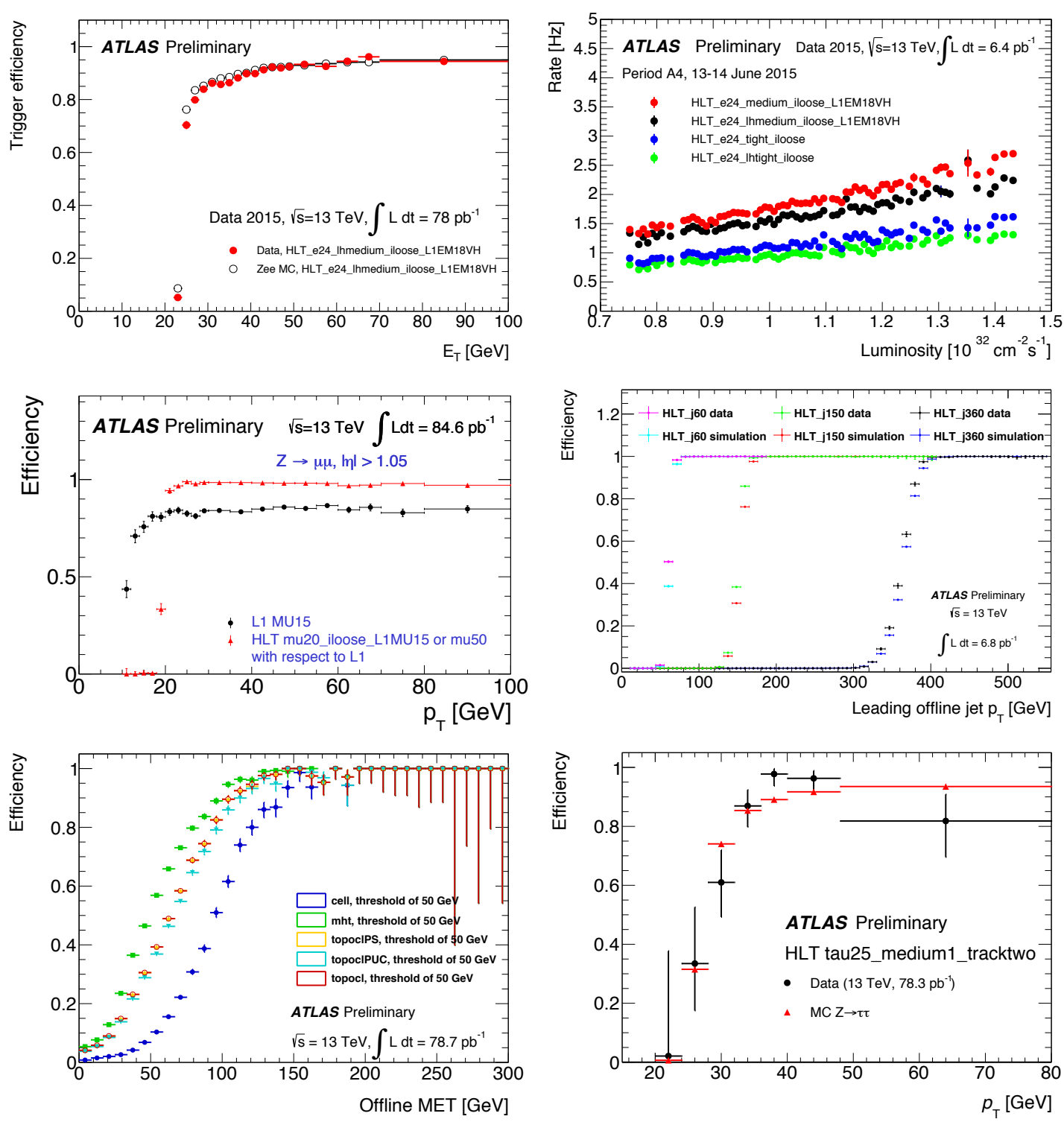

Figure 2: The performance of a selection of main physics triggers in early Run-2 is shown: The efficiency of single electron triggers and their corresponding trigger rates (top panel), the efficiency of single muon triggers (middle left), single jet triggers (middle right), missing transverse energy triggers (bottom left), and single tau lepton triggers (bottom right). Details are given in the text.

HLT_mu20_iloose_L1MU15 trigger is seeded by the L1_MU15 trigger and is required to satisfy a $20 \mathrm{GeV}$ HLT threshold and to pass a loose track isolation selection computed using inner detector tracks. The HLT_mu50 trigger is seeded by the L1_MU20 trigger and is required to satisfy a 50 GeV HLT threshold. The HLT efficiency for the OR of the two HLT triggers is reported relative to the OR of the two L1 seeds. The efficiency is measured using a tag-and-probe method with $Z \rightarrow \mu \mu$ candidates in $13 \mathrm{TeV}$ data with $50 \mathrm{~ns} \mathrm{LHC}$ bunch spacing, with no background subtraction applied. Only statistical data uncertainties are shown. More details can be found in [reference PoS LP15 proceedings: "The ATLAS Muon Trigger Performance in Run I and Initial Run II Performance" 
by Rafal Bielski].

Fig. 2 (middle right) shows a comparison of per-event trigger efficiency turn-on curves between data and MC simulation for three typical jet trigger thresholds used during early Run-2 data-taking. The HLT jets are formed from topo-clusters calibrated to the electromagnetic energy scale. The HLT jets are then calibrated to the hadronic scale by first applying a jet-by-jet area subtraction procedure followed by a jet energy scale weighting that is dependent on the HLT jet transverse momentum and pseudorapidity. Each efficiency is determined using events retained with a lower threshold trigger that is found to be fully efficient in the phase space of interest. More details can be found in [reference PoS LP15 proceedings: "The ATLAS Jet Trigger at 13 TeV" by Giulio Grossi].

Fig. 2 (bottom left) shows missing transverse momentum trigger efficiency turn-on curves for a threshold of $50 \mathrm{GeV}$ as a function of the offline reconstructed missing transverse energy for a number of different algorithms that are currently being commissioned: A 2-sided 2-sigma noise suppression cell-based algorithm (denoted cell), an algorithm based on topological calorimeter clusters (topoclusters) [4] with no further corrections (denoted topocl), a topocluster-based algorithm with hadronic calibration which subtracts average pseudorapidity-dependent pile-up energy per unit area (denoted topoclPS), a topocluster-based algorithm with hadronic calibration that requires at least one jet and performs a fit in which the transverse energy originating in pile-up collisions is constrained to zero (denoted topoclPUC), and an algorithm based on the sum of the trigger jet momenta (denoted mht). All algorithms reach the plateau efficiency at approximately $150 \mathrm{GeV}$ and studies are currently in progress to determine the default choice for future data-taking. More details can be found in [reference PoS LP15 proceedings: "The ATLAS Transverse Momentum Trigger Evolution at the LHC towards Run II" by Antonia Struebig].

Fig. 2 (bottom right) shows the combined Level-1 and HLT tau trigger efficiency measured in data and compared to simulation, with respect to offline reconstructed tau candidates with transverse momentum above $20 \mathrm{GeV}$, one or three tracks and passing the offline "medium" identification criteria. The corresponding trigger tau candidate is required to have a transverse momentum of at least $25 \mathrm{GeV}$, between one and three tracks and pass the trigger "medium" identification. The trigger efficiency is measured with the first $13 \mathrm{TeV}$ collisions data in an enriched sample of $Z \rightarrow \tau \tau \rightarrow \mu \tau^{\text {had }}$, where the $\tau$-lepton decays hadronically. The efficiency is plotted as function of the transverse momentum of the offline tau candidate. Only statistical uncertainties are shown. More details can be found in [reference PoS LP15 proceedings: "The ATLAS Hadronic Tau Trigger Run I and Initial Run II Strategy and Performance" by Mark Pickering].

\section{Conclusion}

The ATLAS trigger system has been upgraded significantly during the LHC shutdown period to sustain the more difficult data-taking conditions in Run-2 and to select the physics processes of interest with high efficiency. At L1 these new features include upgrades to the L1 calorimeter and muon systems, the L1 central trigger processor, and a conceptually new L1 topological subsystem. At the HLT the main structural change is the merging of the previously two-level system into a single event processing farm and a major redesign of the trigger reconstruction algorithms. 
The upgraded ATLAS trigger system is currently being commissioned and first performance results of the main physics triggers in this early phase of Run-2 data-taking have been presented.

\section{References}

[1] ATLAS Collaboration JINST 3 (2008) S08003.

[2] L. Evans and P. Bryant, LHC Machine, JINST 3 (2008) S08001.

[3] ATLAS Collaboration, Technical Design Report for the Phase-I Upgrade of the ATLAS TDAQ System, CERN-LHCC-2013-018, ATLAS-TDR-023 (2013).

https://cds.cern.ch/record/1602235.

[4] W. Lampl et al., Calorimeter clustering algorithms: Description and performance, ATL-LARG-PUB-2008-002 (2008) . https : / cds . cern.ch/record/1099735. 